\title{
Umweltberichterstattung: Eine Zukunftsaufgabe für Unternehmen
}

\author{
Rund 200 deutsche Unternehmen haben mittlerweile einen Umweltbericht oder \\ eine Umwelterklärung verfaßt. Ein umfassender Vergleich zeigte, daß wesent- \\ liche Umweltfragen noch zu wenig deutlich herausgearbeitet werden, Rohstoff- \\ und Produktfragen zu wenig berücksichtigt werden und die Umweltziele des \\ Unternehmens oft nicht überprüfbar sind.
}

$\mathrm{W}$ Von Klaus Fichter und Jens Clausen as leisten sich Unternehmen in puncto Umweltverbrauch und was leisten sie an Umweltschutz? Während bis 1990 weltweit noch nicht einmal zehn Unternehmen einen umfassenden Bericht über ihre Aktivitäten und Ergebnisse im Umweltschutz vorgelegt hatten, ist die Zahl von Umweltberichten und Umwelterklärungen deutscher Unternehmen mittlerweile auf rund 200 angestiegen. Deutschland gehört damit neben USA, Kanada und Großbritannien zu den Ländern mit den berichterstattungsfreudigsten Unternehmen. Die Gesamtzahl von Unternehmen, die Umweltberichte veröffentlichen, dürfte zum gegenwärtigen Zeitpunkt weltweit die 1000er-Grenze noch nicht überschritten haben - gemessen an der Gesamtzahl von Unternehmen eine nach wie vor verschwindend kleine Zahl. Umweltberichterstattung ist noch keine gängige Praxis und eine dialog- und problemorientierte Kommunikation über Umweltfragen schon gar nicht.

Mehr Transparenz über die von Unternehmen und ihren Produkten ausgehenden Umweltbelastungen und der zu ihrer Vermeidung und Verminderung ergriffenen Maßnahmen fordern nicht nur Umweltverbände und -wissenschaftler. Auch die eigenen Mitarbeiter, Kunden/Lieferanten, die Nachbarn oder auch Banken und Versicherungen verlangen zunehmend mehr Umweltinformationen von Unternehmen und zwar nicht nur „die guten“, sondern auch ,die schlechten“ Die aktive Kommunikation über Umweltfragen stellt sowohl eine Notwendigkeit wie auch eine Chance für Unternehmen dar.

\section{Der Boom kam mit dem Öko-Audit}

Dabei ist Umweltberichterstattung von Unternehmen nicht nur gesellschaftlich und moralisch, sondern auch betriebswirtschaftlich geboten. Mit den steigenden Anforderungen von Endverbrauchern, Industriekunden, Behörden, Banken und Versicherungen an die Umweltschutzleistung eines Unternehmens gewinnt die Umweltberichterstattung und -kommunikation an Bedeutung für die Wettbewerbsund Innovationsfähigkeit.

Nachdem die Zahl von Umweltberichten deutscher Unternehmen seit Ende der 80er Jahre stetig aber langsam angestiegen ist, ist seit Sommer 1995 ein rasanter Anstieg zu beobachten. Dies ist auf die EG-Öko-Audit-Verordnung zurückzuführen. Seit September 1995 können sich gewerbliche Unternehmen freiwillig einer Umweltprïfung unterziehen. Die teilnehmenden Unternehmen verpflichten sich dabei, die Öffentlichkeit regelmäßig über den Stand der Umweltschutzarbeit zu informieren. Dies erfolgt durch die sogenannte „Umwelterklärung“ die mindestens alle drei Jahre zu veröffentlichen ist. Bei der Umwelterklärung handelt es sich um einen Standort-Umweltbericht, da sich die Umweltbetriebsprïfung und die Zertifzierung nach der EG-Öko-Audit-Verordnung zumindest vorläufig immer auf Standorte beziehen. Bis April 1996 waren in Deutschland bereits rund 150 Standorte zertifiziert und registriert.

Die Erfahrungen der Pionierunternehmen im Bereich Umweltberichterstattung haben gezeigt, daß Umweltberichte und Umwelterklärungen einen vielfältigen Nutzen entfalten, vorausgesetzt, sie werden als Chance und als Teil eines offenen und umfassenden Dialoges mit den Anspruchgsgruppen eines Unternehmens verstanden.

\section{Qualität deutscher Berichte und Erklärungen}

Eine von future e.V. und dem Institut für ökologische Wirtschaftsforschung gGmbH (IÖW) durchgeführte Analyse und Bewertung von 97

\section{Umweltschutz als Chance}

\section{Praxishilfen für Manager}

Reihe: Ökologische Unternehmensführung Herausgegeben von

Prof. Dr. Siegmar Bornemann Umweltakademie Fresenius, Dortmund Prof. Dr. Reinhard Pfriem Universität Oldenburg

Prof. Dr. Volker Stahlmann Fachhochschule Nürnberg

Prof. Dr. Bernd Wagner

Universität Augsburg

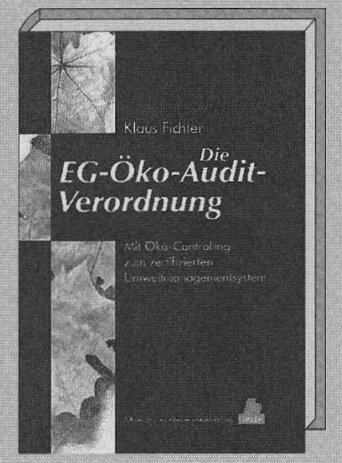

Klaus Fichter (Hrsg.)

Die EG-Öko-Audit-Verordnung

Mit Öko-Controlling zum zertifizierten Umweltmanagementsystem. 381 Seiten

68 Bilder. 1995. Gebunden DM 68,ISBN 3-446-18248-9

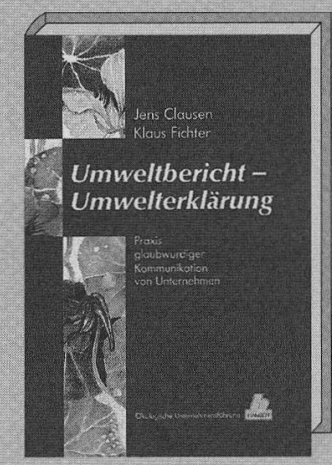

Jens Clausen/Klaus Fichter Umweltbericht-Umwelterklärung Praxis glaubwürdiger Kommunikation von Unternehmen. 245 Seiten, 52 Bilder. 1996. Gebunden DM 58,-. ISBN 3-446-18247-0

Carl Hanser Verlag Postfach 8604 20, 81631 München Tel. (0 89) 998 30-0, Fax (0 89) 981264 


\section{Tabelle 1: Die zwölf besten deutschen Umweltberichte und Umwelterklärungen, die bei dem vergleichenden Ranking durch das lÖW ermittelt wurden. Die im Ranking maximal zu erreichende Punkłzahl beträgt $\mathbf{5 0}$.}

\begin{tabular}{|c|c|c|c|c|c|}
\hline Rang & Unternehmen & Bericht & Branche & Erreichte Punkizahl & Zertifizierte Umwelterklärung \\
\hline 1 & Neumarkter Lammsbräu & Öko-Controlling-Bericht 1994 & Emöhrung & 425 & nein \\
\hline 2 & Kunert & Ökobericht 1994/95 & Textil & 414 & nein \\
\hline 3 & Sedus Stoll & Umwelterklärung Waldshut & Möbel & 382 & jo \\
\hline 4 & Neckermann & Umwelterklärung 7995 & Hondel & 379 & nein \\
\hline 5 & Siegsdorfer Petrusquelle & Unweltbericht 93/94 & Emährung & 374 & nein \\
\hline 6 & Mineralbrunnen Bad Brückenau & Umweltbericht 1993/94 & Enährung & 370 & nein \\
\hline 7 & Märkisches Landbrot & Umwelterklärung & Emährung & 356 & jo \\
\hline 8 & Boufritz Vollwerthous & Unwellbericht mit Ökobilanz 94/95 & Bou & 352 & nein \\
\hline 9 & Kraft Jocobs Suchard & Umweltbericht 1991-1995 & Enährung & 343 & nein \\
\hline 10 & Augsburger Kammgarn-Spinnerei & Umweltbericht 1994 & Textil & 342 & nein \\
\hline 11 & Hipp & Unwelthericht und Umwelterklärung & Enährung & 340 & io \\
\hline 12 & Bosch-Siemens Hausgeräte & Umweltbericht 1994/95 & Housgeräte & 336 & nein \\
\hline
\end{tabular}

Quelle: Clousen, J; Fichter, K. (1996): Die Qualität von Umweltberichten: Ranking 19, Berlin.

Umweltberichten und Umwelterklärungen deutscher Unternehmen, die 1995 veröffentlicht wurden, zeigt, daß die Berichte in den vergangenen Jahren in ihrem Aufbau systematischer und generell vollständiger geworden sind. Die Berichte spiegeln wider, daß sich das betriebliche Umweltmanagement und die Datenlage verbessert haben.

Die bei der vergleichenden Bewertung (Ranking 1995) ermittelten zwölf besten deutschen Umweltberichte und Umwelterklärungen sind in Tabelle 1 dargestellt. Das Ranking 1995 hat gezeigt, daß es noch eine Reihe typischer Schwachpunkte gibt, die von denjenigen, die Umweltberichte und Umwelterklärungen verfassen und gestalten, unbedingt berücksichtigt werden sollten.

\section{- Wesentliche Umweltfragen deutlich machen}

Die mangelnde Analyse und Bewertung von Daten führt dazu, daß es die wenigsten Umweltberichte leisten, die für das Unternehmen wichtigsten Umweltfragen herauszuarbeiten und im Überblick darzustellen. Oft werden wesentliche Umweltfragen, z.B. produktbezogene Aspekte des Umweltschutzes vergessen und alle Umweltaspekte in gleicher Tiefe oder Breite dargestellt. Angesichts der Menge und Vielfalt an Umweltinformationen ist dies nicht wünschenswert! Zur Herausarbeitung wesentlicher Umweltfragen bedarf es der Anstrengung auf betrieblicher wie auf Branchenebene.
Im Mittelpunkt der meisten Umweltberichte, insbesondere der Chemieindustrie, dominieren nach wie vor standortbezogene Umweltaspekte, und hier in erster Linie Emissionsfragen (Luft-, Wasseremissionen, Abfälle). Produkte bzw. Dienstleistungen als eigentlicher Gegenstand der betrieblichen Leistungserstellung und insbesondere die vor- und nachgelagerten Phasen des ökologischen Produktlebensweges sind noch weitgehend unterbelichtet. Gerade die chemische Industrie hat hier noch erheblichen Verbesserungsbedarf. Sieht man von der Umwelterklärung 1995 der Ciba Additive $\mathrm{GmbH}$ einmal $\mathrm{ab}$, geht kein Umweltbericht der Chemieindustrie in nennenswerter Weise auf den Verbrauch von Rohstoffen ein

Das Ranking 1995 zeigt, in deutschen Unternehmen wird noch zu wenig Wert auf klare und überprüfbare Zielsetzungen im Umweltmanagement gelegt. Wenngleich sich hier eine Verbesserung beobachten läßt, zeigt sich, daß bei deutschen Unternehmen nach wie vor ein eher daten-orientiertes Umweltmanagement betrieben wird. Noch fehlen Mut und Erfahrung zum Setzen anspruchsvoller und überprüfbarer Umweltziele.

Zwar werden in den Umwelterklärungen Fristen genannt, allerdings bleiben die Ziele allgemein und kaum bzw. nicht überprüfbar. Auch werden Umweltziele bislang ohne Blick für kommunale, nationale und internationale Zielsetzungen im Umweltschutz formuliert.

\section{Teil eines Dialoges}

Insbesondere die Umweltberichte mittelständischer Unternehmen und die Standortberichte von Konzernen werden noch zu wenig als Teil eines Kommunikationsprozesses begriffen. Das direkte Ansprechen der Zielgruppen fehlt in der Regel. Umwelterklärungen werden zu wenig als Instrument der Öffentlichkeitsarbeit und $\mathrm{zu}$ wenig als Chance für Dialog und Kooperationen genutzt. Vorbildlich ist hier die Umwelterklärung des Berliner Backwarenherstellers Märkisches Landbrot. Dort werden die Umweltaktivitäten mit Verbrauchern, Einzelhandel und Lieferanten anschaulich beschrieben. Auch mehrere Mitarbeiter/innen kommen in der Umwelterklärung zu Wort.

Die Schwächen bei der Darstellung bisheriger und zukünftiger Aktivitäten mit Zielgruppen korrespondiert mit der insgesamt mangelhaften sprachlichen und optischen Gestaltung der Berichte. In puncto kommunikative Qualität besteht also ein erheblicher Verbesserungsbedarf. Das Niveau von Geschäftsberichten wird diesbeziiglich noch bei weitem nicht erreicht.

\section{Die Referenten}

Klaus Fichter ist wissenschuffiticher Mitarbeiter am Insitut für Ökologische Wirtschaftsforschung in Berlin, Jens Clausen ist wissenschafflicher Mitarbeiter am IÖW in Hannover

Kontakr: Tel. (030) 884 594-20, Fax 8825439 
(c) 20I0 Authors; licensee IÖW and oekom verlag. This is an article distributed under the terms of the Creative Commons Attribution Non-Commercial No Derivates License (http://creativecommons.org/licenses/by-nc-nd/3.o/), which permits unrestricted use, distribution, and reproduction in any medium, provided the original work is properly cited. 